\title{
ГИПОПАРАТИРЕОЗ В МОСКОВСКОЙ ОБЛАСТИ: СТАРТ ВЕДЕНИЯ ВСЕРОССИЙСКОГО РЕГИСТРА
}

\author{
Красулина К.А., Крюкова И.В., Древаль А.В., Полякова Е.Ю.
}

ГБУЗ МО «Московский областной научно-исследовательский клинический институт им. М.Ф. Владимирского», 2. Москва

ЦЕЛЬ: оценить результаты начала ведения регистра гипопаратиреоза в Московской области (МО) в рамках Всероссийской базы данных онлайн-версии Государственного регистра больных с гипопаратиpeозом.

МАТЕРИАЛЫ И МЕТОДЫ: проведен анализ регистра пациентов с гипопаратиреозом в МО в рамках всероссийского Государственного регистра больных гипопаратиреозом, который ведется на базе ГБУз МО МОНИКИ им М.Ф. Владимирского с конца 2020 г. Критерием включения являлся лабораторно подтвержденный стойки гипопаратиреоз: паратгормон (ПТГ) ниже нижней границы референсных значений (15-65 пг/мл) и общий кальций крови менее 2,1 ммоль/л. Критерии исключения: гипокальциемия, не связанная со снижением ПТГ. Данные оценивались на июнь 2021 г.

РЕЗУЛЬТАТЫ И ОБСУЖДЕНИЕ: всего в регистр внесены данные 37 пациентов с гипопаратиреозом: 32 женщины и 5 мужчин. Средний возраст составил 54,8 года (44,8 года у мужчин и 56,4 года у женщин), средний возраст постановки диагноза - 49,97 года (у мужчин - 43,6 года, у женщин - 51 год). Среди них было 35 (94,6\%) человек с послеоперационным (31 женщина и 4 мужчины) гипопаратиреозом и 2 (1 женщина, 1 мужчина) - с идиопатическим (5,4\%). В 23 (65,7\%) случаях в анамнезе была тиреоидэктомия, в $6(17,1 \%)$ - паратиреоидэктомия, в $4(11,4 \%)$ - сочетанная операция, в 1 - гемитиреоидэктомия (2,9\%) и в 1 - оперативное лечение флегмоны шеи (2,9\%).

Максимальная выявляемость гипопаратиреоза была в 2019 г. и составила 7 случаев (5 женщин, 2 мужчин). В 2018 г. заболевание диагностировано у 5 женщин и 1 мужчины. В 2020 г. диагноз установлен 4 женщинам, в 2014 и 2017 гг. - 3 женщинам ежегодно, а также 2 мужчинам и 1 женщине в 1-й половине 2021 г. В 2012, 2015 и 2016 гг. выявлялись по 2 и по 1 пациенту в 1999, 2003, 2009, 2010, 2013 гг. В другие годы не было зарегистрировано новых случаев гипопаратиреоза.

На момент оценки гипопаратиреоз был компенсирован у 11 (29,7\%) пациентов (10 женщин), субкомпенсирован - у 10 (27,1\%), из них 8 женщин, декомпенсирован - у 16 (43,2\%), 14 женщин.

Симптомы гипокальциемии отмечались в виде: судорог у 43,2\% больных, слабости - у 32,4\%, парестезий - у 21,6\%. Боли в костях и суставах беспокоили 18,9\% больных. У 8,1\% диагностирована катаракта, у 5,4\% - нефролитиаз. Кальцификация структур головного мозга выявлена у 2 человек по результатам КТ. Причем, у мужчины гипопаратиреоз, предположительно, был длительно декомпенсирован (диагноз поставлен в 2016 г., а тиреоидэктомия, послужившая причиной, проведена в 1991 г.), у женщины диагностирован идиопатический гипопаратиреоз, истинная длительность которого неизвестна (первые проявления в виде судорог отмечены в 2012 г., диагноз поставлен в 2018 г.).

Средний уровень ПТГ у больных (n=37) составил 3,68 пг/мл. При 1 визите средний уровень кальция

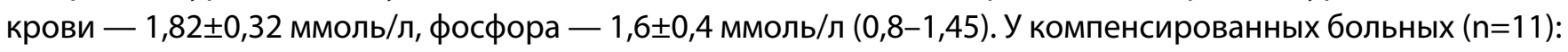

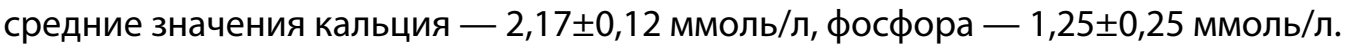

ВЫВОДЫ: ведение регистра пациентов с гипопаратиреозом позволяет оценить его распространенность и заболеваемость в популяции, степень компенсации, частоту осложнений, а также потребность в заместительной терапии. В последние годы в МО отмечается увеличение числа новых случаев данного редкого заболевания, что свидетельствует о его лучшей выявляемости. 